\title{
Radiative Decays of Heavy Hadrons From Light-Cone QCD Sum Rules in the Leading Order of HQET
}

\author{
Shi-Lin Zhu and Yuan-Ben Dai \\ Institute of Theoretical Physics, Academia Sinica, P.O.Box 2735, Beijing 100080, China
}

\begin{abstract}
The radiative decays of heavy baryons and lowest three doublets of heavy mesons are studied with the light cone QCD sum rules in the leading order of heavy quark effective theory.
\end{abstract}

PACS number: 12.39.Hg, 13.40.Hq, 12.38.Lg

Keywords: HQET, radiative decay, light cone QCD sum rule

\section{Introduction}

Heavy quark effective theory (HQET) [1] provides a framework to study the heavy hadron spectra and transition amplitude with the systematic expansion in terms of $1 / m_{Q}$. Using the light cone photon wave function (PWF), radiative decay processes like $B \rightarrow l \nu \gamma$, $B \rightarrow \rho \gamma$ have been studied [2, 3, 4, 5, 6] with QCD sum rules (QSR) [7]. Recently similar approach was employed to analyze the couplings of pions with heavy hadrons [8]. In this work we will study the radiative decays of heavy baryons and lowest three doublets of heavy mesons using the light cone QSR (LCQSR) [9] in the leading order of HQET. With LCQSR the continuum and excited states contribution is subtracted more cleanly, which is in contrast with the analysis of meson radiative decays using the external field method in QSR [10]. The LCQSRs for the radiative decays of heavy baryons are presented in section 2. Section 3 discusses radiative decays of lowest three doublets of heavy mesons. The following section is a discussion of the parameters and the photon wave functions. The last section is the numerical analysis and a short summary.

\section{Radiative decays of heavy baryons}

We first introduce the interpolating currents for the heavy baryons:

$$
\begin{gathered}
\eta_{\Lambda}(x)=\epsilon_{a b c}\left[u^{a T}(x) C \gamma_{5} d^{b}(x)\right] h_{v}^{c}(x), \\
\eta_{\Sigma^{+}}(x)=\epsilon_{a b c}\left[u^{a T}(x) C \gamma_{\mu} d^{b}(x)\right] \gamma_{t}^{\mu} \gamma_{5} h_{v}^{c}(x),
\end{gathered}
$$




$$
\eta_{\Sigma^{++*}}^{\mu}(x)=\epsilon_{a b c}\left[u^{a T}(x) C \gamma_{\nu} u^{b}(x)\right]\left(-g_{t}^{\mu \nu}+\frac{1}{3} \gamma_{t}^{\mu} \gamma_{t}^{\nu}\right) h_{v}^{c}(x)
$$

where $a, b, c$ is the color index, $u(x), d(x), h_{v}(x)$ is the up, down and heavy quark fields, $T$ denotes the transpose, $C$ is the charge conjugate matrix, $g_{t}^{\mu \nu}=g^{\mu \nu}-v^{\mu} v^{\nu}, \gamma_{t}^{\mu}=\gamma_{\mu}-\hat{v} v^{\mu}$, and $v^{\mu}$ is the velocity of the heavy hadron.

The overlap amplititudes of the interpolating currents with the heavy baryons is defined as:

$$
\begin{aligned}
\left\langle 0\left|\eta_{\Lambda}\right| \Lambda\right\rangle & =f_{\Lambda} u_{\Lambda}, \\
\left\langle 0\left|\eta_{\Sigma}\right| \Sigma\right\rangle & =f_{\Sigma} u_{\Sigma}, \\
\left\langle 0\left|\eta_{\Sigma^{*}}^{\mu}\right| \Sigma^{*}\right\rangle & =\frac{f_{\Sigma^{*}}}{\sqrt{3}} u_{\Sigma^{*}}^{\mu},
\end{aligned}
$$

where $u_{\Sigma^{*}}^{\mu}$ is the Rarita-Schwinger spinor in HQET. In the leading order of HQET, $f_{\Sigma}=f_{\Sigma^{*}}$ [11.

The coupling constants $\eta_{i}$ are defined through the following amplitudes:

$$
\begin{gathered}
M\left(\Sigma_{c} \rightarrow \Lambda_{c} \gamma\right)=i e \eta_{1} \bar{u}_{\Lambda_{c}} \sigma^{\mu \nu} q_{\mu} e_{\nu} u_{\Sigma_{c}}, \\
M\left(\Sigma_{c}^{*} \rightarrow \Lambda_{c} \gamma\right)=i e \eta_{2} \epsilon_{\mu \nu \alpha \beta} \bar{u}_{\Lambda_{c}} \gamma^{\nu} q^{\alpha} e^{\beta} u_{\Sigma_{c}^{*}}^{\mu}, \\
M\left(\Sigma_{c}^{*} \rightarrow \Sigma_{c} \gamma\right)=i e \eta_{3} \epsilon_{\mu \nu \alpha \beta} \bar{u}_{\Sigma_{c}} \gamma^{\nu} q^{\alpha} e^{\beta} u_{\Sigma_{c}^{*}}^{\mu},
\end{gathered}
$$

where $e_{\mu}$ and $q_{\mu}$ are the photon polarization vector and momentum respectively, $e$ is the charge unit.

In order to derive the sum rules for the coupling constants we consider the correlator

$$
\begin{gathered}
\int d^{4} x e^{-i k \cdot x}\left\langle\gamma(q)\left|T\left(\eta_{\Sigma}(0) \bar{\eta}_{\Lambda}(x)\right)\right| 0\right\rangle=e \frac{1+\hat{v}}{2} \gamma_{t}^{\mu} \gamma_{5} \epsilon_{\mu \alpha \beta \sigma} e^{\alpha} q^{\beta} v^{\sigma} G_{\Sigma, \Lambda}\left(\omega, \omega^{\prime}\right), \\
\int d^{4} x e^{-i k \cdot x}\left\langle\gamma(q)\left|T\left(\eta_{\Sigma^{*}}^{\mu}(0) \bar{\eta}_{\Lambda}(x)\right)\right| 0\right\rangle= \\
e\left(e_{\alpha} q_{\nu}-e_{\nu} q_{\alpha}\right) \frac{1+\hat{v}}{2}\left(-g_{t}^{\mu \nu}+\frac{1}{3} \gamma_{t}^{\mu} \gamma_{t}^{\nu}\right) \epsilon_{\nu \alpha \beta \sigma} e^{\alpha} q^{\beta} v^{\sigma} G_{\Sigma^{*}, \Lambda}\left(\omega, \omega^{\prime}\right) \\
\int d^{4} x e^{-i k \cdot x}\left\langle\gamma(q)\left|T\left(\eta_{\Sigma^{*}}^{\mu}(0) \bar{\eta}_{\Sigma}(x)\right)\right| 0\right\rangle= \\
e \frac{1+\hat{v}}{2} \gamma_{t}^{\alpha} \gamma_{5}\left(-g_{t}^{\mu \nu}+\frac{1}{3} \gamma_{t}^{\mu} \gamma_{t}^{\nu}\right)\left(e_{\alpha} q_{\nu}-e_{\nu} q_{\alpha}\right) G_{\Sigma^{*}, \Sigma}\left(\omega, \omega^{\prime}\right)
\end{gathered}
$$

where $k^{\prime}=k-q, q_{\mu}^{t}=q_{\mu}-(q \cdot v) v_{\mu}, \omega=2 v \cdot k, \omega^{\prime}=2 v \cdot k^{\prime}$ and $q^{2}=0$.

Let us first consider the functions $G_{\Sigma, \Lambda}\left(\omega, \omega^{\prime}\right)$ etc in (10)-(12). As functions of two variables, they have the following pole terms from double dispersion relation

$$
\begin{gathered}
\frac{-4 i \eta_{1} f_{\Sigma} f_{\Lambda}}{\left(2 \bar{\Lambda}_{\Sigma}-\omega^{\prime}\right)\left(2 \bar{\Lambda}_{\Lambda}-\omega\right)}+\frac{c}{2 \bar{\Lambda}_{\Sigma}-\omega^{\prime}}+\frac{c^{\prime}}{2 \bar{\Lambda}_{\Lambda}-\omega}, \\
\frac{-4 i \eta_{2}}{\sqrt{3}} \frac{f_{\Sigma^{*}} f_{\Lambda}}{\left(2 \bar{\Lambda}_{\Sigma^{*}}-\omega^{\prime}\right)\left(2 \bar{\Lambda}_{\Lambda}-\omega\right)}+\frac{c}{2 \bar{\Lambda}_{\Sigma^{*}}-\omega^{\prime}}+\frac{c^{\prime}}{2 \bar{\Lambda}_{\Lambda}-\omega},
\end{gathered}
$$




$$
\frac{4 \eta_{3}}{\sqrt{3}} \frac{f_{\Sigma^{*}} f_{\Sigma}}{\left(2 \bar{\Lambda}_{\Sigma^{*}}-\omega^{\prime}\right)\left(2 \bar{\Lambda}_{\Sigma}-\omega\right)}+\frac{c}{2 \bar{\Lambda}_{\Sigma^{*}}-\omega^{\prime}}+\frac{c^{\prime}}{2 \bar{\Lambda}_{\Sigma}-\omega},
$$

where $f_{\Sigma^{*}}$ etc are constants defined in (44)-(6), $\bar{\Lambda}_{\Sigma^{*}}=m_{\Sigma^{*}}-m_{Q}$.

Keeping the two particle component of the photon wave function, the expression for $G_{\Sigma^{*}, \Lambda}\left(\omega, \omega^{\prime}\right)$ with the tensor structure reads

$$
\begin{aligned}
-2 \int_{0}^{\infty} d t \int d x e^{-i k x} \delta(-x-v t) & \operatorname{Tr}\left\{\left[C\langle\gamma(q)|u(0) \bar{u}(x)| 0\rangle^{T} C \gamma_{\mu} i S(-x) \gamma_{5}\right]\right. \\
+ & {\left.\left.\left[C i S^{T}(-x) C \gamma_{\mu}\right]\langle\gamma(q)|u(0) \bar{u}(x)| 0\rangle \gamma_{5}\right]\right\}, }
\end{aligned}
$$

where $i S(-x)$ is the full light quark propagator with both perturbative term and contribution from vacuum fields.

$$
\begin{aligned}
i S(x) & =\langle 0|T[q(x), \bar{q}(0)]| 0\rangle=i \frac{\hat{x}}{2 \pi^{2} x^{4}}-\frac{\langle\bar{q} q\rangle}{12}-\frac{x^{2}}{192}\left\langle\bar{q} g_{s} \sigma \cdot G q\right\rangle \\
& -i g_{s} \frac{1}{16 \pi^{2}} \int_{0}^{1} d u\left\{\frac{\hat{x}}{x^{2}} \sigma \cdot G(u x)-4 i u \frac{x_{\mu}}{x^{2}} G^{\mu \nu}(u x) \gamma_{\nu}\right\}+\cdots .
\end{aligned}
$$

The light cone two-particle photon wave functions are [2]:

$$
\begin{gathered}
<\gamma(q)\left|\bar{q}(x) \sigma_{\mu \nu} q(0)\right| 0>=i e_{q} e\langle\bar{q} q\rangle \int_{0}^{1} d u e^{i u q x}\left\{\left(e_{\mu} q_{\nu}-e_{\nu} q_{\mu}\right)\left[\chi \phi(u)+x^{2} g_{1}(u)\right]\right. \\
\left.+\left[(q x)\left(e_{\mu} x_{\nu}-e_{\nu} x_{\mu}\right)+(e x)\left(x_{\mu} q_{\nu}-x_{\nu} q_{\mu}\right)-x^{2}\left(e_{\mu} q_{\nu}-e_{\nu} q_{\mu}\right)\right] g_{2}(u)\right\} \\
<\gamma(q)\left|\bar{q}(x) \gamma_{\mu} \gamma_{5} q(0)\right| 0>=\frac{f}{4} e_{q} e \epsilon_{\mu \nu \rho \sigma} e^{\nu} q^{\rho} x^{\sigma} \int_{0}^{1} d u e^{i u q x} \psi(u) .
\end{gathered}
$$

Due to the choice of the gauge $x^{\mu} A_{\mu}(x)=0$, the path-ordered gauge factor

$P \exp \left(i g_{s} \int_{0}^{1} d u x^{\mu} A_{\mu}(u x)\right)$ has been omitted. The $\phi(u), \psi(u)$ is associated with the leading twist two photon wave function, while $g_{1}(u)$ and $g_{2}(u)$ are twist-4 PWFs. All these PWFs are normalized to unity, $\int_{0}^{1} d u f(u)=1$.

Expressing (16) with the photon wave functions, we arrive at:

$$
\begin{gathered}
G_{\Sigma^{*}, \Lambda}\left(\omega, \omega^{\prime}\right)=-\left(e_{u}-e_{d}\right) \int_{0}^{\infty} d t \int_{0}^{1} d u e^{i(1-u) \frac{\omega t}{2}} e^{i u \frac{\omega^{\prime} t}{2}}\left\{\langle \overline { q } q \rangle \left[\frac{1}{\pi^{2} t^{3}} \chi \phi(u)\right.\right. \\
\left.\left.+\frac{1}{\pi^{2} t}\left(g_{1}(u)-g_{2}(u)\right)\right]+\frac{f}{24} \psi(u) t\left(\langle\bar{q} q\rangle+\frac{t^{2}}{16}\left\langle\bar{q} g_{s} \sigma \cdot G q\right\rangle\right)\right\}+\cdots .
\end{gathered}
$$

Similarly we have,

$$
\begin{gathered}
G_{\Sigma, \Lambda}\left(\omega, \omega^{\prime}\right)=G_{\Sigma^{*}, \Lambda}\left(\omega, \omega^{\prime}\right), \\
G_{\Sigma^{*}, \Sigma}\left(\omega, \omega^{\prime}\right)=\left(e_{u}+e_{d}\right) \int_{0}^{\infty} d t \int_{0}^{1} d u e^{i(1-u) \frac{\omega t}{2}} e^{i u \frac{\omega^{\prime} t}{2}}\left\{\frac{f}{4 \pi^{2} t^{2}} \psi(u)\right. \\
+\frac{\langle\bar{q} q\rangle}{6}\left(\langle\bar{q} q\rangle+\frac{t^{2}}{16}\left\langle\bar{q} g_{s} \sigma \cdot G q\right\rangle\right)\left[\chi \phi(u)+t^{2}\left(g_{1}(u)-g_{2}(u)\right]\right\}+\cdots,
\end{gathered}
$$

where $\langle\bar{q} q\rangle=-(225 \mathrm{MeV})^{3},\left\langle\bar{q} g_{s} \sigma \cdot G q\right\rangle=m_{0}^{2}\langle\bar{q} q\rangle, m_{0}^{2}=0.8 \mathrm{GeV}^{2}$. For large Euclidean values of $\omega$ and $\omega^{\prime}$ this integral is dominated by the region of small $t$, therefore it can be approximated by the first a few terms. 
After Wick rotations and making double Borel transformation with the variables $\omega$ and $\omega^{\prime}$ the single-pole terms in (13)-(15) are eliminated. Subtracting the continuum contribution which is modeled by the dispersion integral in the region $\omega, \omega^{\prime} \geq \omega_{c}$, we arrive at:

$$
\begin{array}{ccc}
\eta_{1} f_{\Sigma} f_{\Lambda}= & -\frac{1}{64 \pi^{4}}\left(e_{u}-e_{d}\right) a e^{\frac{\Lambda_{\Sigma}+\Lambda_{\Lambda}}{T}}\left\{\chi \phi\left(u_{0}\right) T^{4} f_{3}\left(\frac{\omega_{c}}{T}\right)\right. \\
\left.-4\left[g_{1}\left(u_{0}\right)-g_{2}\left(u_{0}\right)\right] T^{2} f_{1}\left(\frac{\omega_{c}}{T}\right)+\frac{2 \pi^{2}}{3} f \psi\left(u_{0}\right)\left(1-\frac{m_{0}^{2}}{4 T^{2}}\right)\right\} \\
\eta_{2} f_{\Sigma^{*}} f_{\Lambda}=\quad \begin{array}{c}
-\frac{\sqrt{3}}{64 \pi^{4}}\left(e_{u}-e_{d}\right) a e^{\frac{\Lambda_{\Sigma^{*}+\Lambda_{\Lambda}}}{T}}\left\{\chi \phi\left(u_{0}\right) T^{4} f_{3}\left(\frac{\omega_{c}}{T}\right)\right. \\
\left.-4\left[g_{1}\left(u_{0}\right)-g_{2}\left(u_{0}\right)\right] T^{2} f_{1}\left(\frac{\omega_{c}}{T}\right)+\frac{2 \pi^{2}}{3} f \psi\left(u_{0}\right)\left(1-\frac{m_{0}^{2}}{4 T^{2}}\right)\right\}
\end{array} \\
\eta_{3} f_{\Sigma^{*}} f_{\Sigma}= & \frac{\sqrt{3}}{32 \pi^{2}}\left(e_{u}+e_{d}\right) e^{\frac{\Lambda_{\Sigma^{*}+\Lambda_{\Sigma}}}{T}}\left\{f \psi\left(u_{0}\right) T^{3} f_{2}\left(\frac{\omega_{c}}{T}\right)\right. \\
\left.-\frac{a^{2}}{6 \pi^{2}}\left(1-\frac{m_{0}^{2}}{4 T^{2}}\right)\left[\chi \phi\left(u_{0}\right) T f_{0}\left(\frac{\omega_{c}}{T}\right)-\frac{4}{T}\left(g_{1}\left(u_{0}\right)-g_{2}\left(u_{0}\right)\right)\right]\right\}
\end{array}
$$

where $f_{n}(x)=1-e^{-x} \sum_{k=0}^{n} \frac{x^{k}}{k !}$ is the factor used to subtract the continuum, $\omega_{c}$ is the continuum threshold. $u_{0}=\frac{T_{1}}{T_{1}+T_{2}}, T \equiv \frac{T_{1} T_{2}}{T_{1}+T_{2}}, T_{1}, T_{2}$ are the Borel parameters $a=$ $-(2 \pi)^{2}\langle\bar{q} q\rangle$. We have used the Borel transformation formula: $\hat{\mathcal{B}}_{\omega}^{T} e^{\alpha \omega}=\delta\left(\alpha-\frac{1}{T}\right)$.

Due to the heavy quark symmetry, $\Lambda_{\Sigma}=\Lambda_{\Sigma^{*}}$ and $f_{\Sigma}=f_{\Sigma^{*}}$ in the limit $m_{Q} \rightarrow \infty$. So from (23) and (24) we have $\eta_{2}=\sqrt{3} \eta_{1}$. For the decays $\Sigma_{c}^{* 0} \rightarrow \Sigma_{c}^{0} \gamma$ and $\Sigma_{c}^{*++} \rightarrow \Sigma_{c}^{++} \gamma$, we need make replacement $\left(e_{u}+e_{d}\right) \rightarrow 2 e_{d}, 2 e_{u}$ in (25).

\section{Radiative decays of heavy mesons}

We shall confine ourselves to the lowest lying three doublets and consider all possible radiative decay processes among them in the leading order of $1 / m_{Q}$ expansion. Denote the doublet $\left(1^{+}, 2^{+}\right)$with $j_{\ell}=3 / 2$ by $\left(B_{1}, B_{2}^{*}\right)$, the doublet $\left(0^{+}, 1^{+}\right)$with $j_{\ell}=1 / 2$ by $\left(B_{0}^{\prime}, B_{1}^{\prime}\right)$ and the doublet $\left(0^{-}, 1^{-}\right)$by $\left(B, B^{*}\right)$.

The interpolating currents are given in [12] as

$$
\begin{aligned}
& J_{1,+, \frac{3}{2}}^{\dagger \alpha}=\sqrt{\frac{3}{4}} \bar{h}_{v} \gamma^{5}(-i)\left(\mathcal{D}_{t}^{\alpha}-\frac{1}{3} \gamma_{t}^{\alpha} \mathcal{D}_{t}\right) q, \\
& J_{2,+, \frac{3}{2}}^{\dagger \alpha_{1}, \alpha_{2}}=\sqrt{\frac{1}{2}} \bar{h}_{v} \frac{(-i)}{2}\left(\gamma_{t}^{\alpha_{1}} \mathcal{D}_{t}^{\alpha_{2}}+\gamma_{t}^{\alpha_{2}} \mathcal{D}_{t}^{\alpha_{1}}-\frac{2}{3} g_{t}^{\alpha_{1} \alpha_{2}} \mathcal{D}_{t}\right) q, \\
& J_{1,-, \frac{1}{2}}^{\dagger \alpha}=\sqrt{\frac{1}{2}} \bar{h}_{v} \gamma_{t}^{\alpha} q, \quad J_{0,-, \frac{1}{2}}^{\dagger \alpha}=\sqrt{\frac{1}{2}} \bar{h}_{v} \gamma_{5} q, \\
& J_{0,+, \frac{1}{2}}^{\dagger}=\frac{1}{\sqrt{2}} \bar{h}_{v} q, \quad J_{1,+, \frac{1}{2}}^{\dagger \alpha}=\frac{1}{\sqrt{2}} \bar{h}_{v} \gamma^{5} \gamma_{t}^{\alpha} q .
\end{aligned}
$$


- $\left(1^{+}, 2^{+}\right) \rightarrow\left(0^{-}, 1^{-}\right)+\gamma$

The decay amplitudes are

$$
\begin{aligned}
& M\left(B_{1} \rightarrow B^{*} \gamma\right)=e_{q} e e^{* \mu} v^{\sigma} \epsilon_{\beta}^{*} \eta_{\alpha}\left\{\left[\epsilon_{\mu \nu \beta \sigma} q_{t}^{\alpha}+(\alpha \leftrightarrow \beta)\right] q_{t}^{\nu} g_{D}^{1}\left(B_{1}, B^{*}\right)\right. \\
+ & {\left.\left[\epsilon_{\mu \nu \beta \sigma}\left(q_{t}^{\alpha} q_{t}^{\nu}-\frac{1}{3} q_{t}^{2} g_{t}^{\alpha \nu}\right)-(\alpha \leftrightarrow \beta)\right] g_{D}^{2}\left(B_{1}, B^{*}\right)+\epsilon_{\mu \alpha \beta \sigma} g_{S}\left(B_{1}, B^{*}\right)\right\} }
\end{aligned}
$$

where the tensor structure associated with $g_{D}^{1}\left(B_{1}, B^{*}\right)$ and $g_{D}^{2}\left(B_{1}, B^{*}\right)$ is symmetric and antisymmetric under the exchange of $(\alpha \leftrightarrow \beta)$ respectively.

$$
\begin{gathered}
M\left(B_{1} \rightarrow B \gamma\right)=e_{q} e e_{\beta}^{*} \eta_{\alpha}\left\{\left(q_{t}^{\alpha} q_{t}^{\beta}-\frac{1}{3} q_{t}^{2} g_{t}^{\alpha \beta}\right) g_{D}\left(B_{1}, B\right)+g_{t}^{\alpha \beta} g_{S}\left(B_{1}, B\right)\right\} \\
M\left(B_{2}^{*} \rightarrow B \gamma\right)=e_{q} e e_{\beta}^{*} \eta_{\alpha_{1} \alpha_{2}} q_{\nu} v_{\sigma}\left[\epsilon^{\beta \nu \alpha_{1} \sigma} q_{t}^{\alpha_{2}}+\left(\alpha_{1} \leftrightarrow \alpha_{2}\right)\right] g_{D}\left(B_{2}^{*}, B\right), \\
M\left(B_{2}^{*} \rightarrow B^{*} \gamma\right)=e_{q} e \eta_{\alpha_{1} \alpha_{2}} \eta_{\beta}^{*}\left\{e_{t}^{\beta}\left(q_{t}^{\alpha_{1}} q_{t}^{\alpha_{2}}-\frac{1}{3} q_{t}^{2} g_{t}^{\alpha_{1} \alpha_{2}}\right) g_{D}^{1}\left(B_{2}^{*}, B^{*}\right)+\left[e _ { t } ^ { \alpha _ { 1 } } \left(q_{t}^{\beta} q_{t}^{\alpha_{2}}\right.\right.\right. \\
\left.\left.\left.-\frac{1}{3} q_{t}^{2} g_{t}^{\beta \alpha_{2}}\right)+\left(\alpha_{1} \leftrightarrow \alpha_{2}\right)\right] g_{D}^{2}\left(B_{2}^{*}, B^{*}\right)+\left[e_{t}^{\alpha_{1}} g_{t}^{\alpha_{2} \beta}+\left(\alpha_{1} \leftrightarrow \alpha_{2}\right)\right] g_{S}\left(B_{2}^{*}, B^{*}\right)\right\}
\end{gathered}
$$

where $\eta_{\mu \nu}, \eta_{\mu}$ and $\epsilon_{\mu}$ are polarization tensors for states $2^{+}, 1^{+}$and $1^{-}$respectively and $e_{q} e$ is the light quark electric charge.

Due to heavy quark symmetry, there exist only two independent coupling constants for the D-wave and S-wave decay respectively. Let $g_{d} \equiv g_{D}\left(B_{2}^{*}, B\right)$ and $g_{s} \equiv-g_{S}\left(B_{2}^{*}, B^{*}\right)$. Then we have:

$$
\begin{gathered}
\frac{\sqrt{6}}{2} g_{S}\left(B_{1}, B^{*}\right)=\frac{\sqrt{6}}{4} g_{S}\left(B_{1}, B\right)=g_{s} \\
\frac{\sqrt{6}}{3} g_{D}^{1}\left(B_{1}, B^{*}\right)=\sqrt{6} g_{D}^{2}\left(B_{1}, B^{*}\right)=\frac{\sqrt{6}}{2} g_{D}\left(B_{1}, B\right) \\
=-\frac{1}{2} g_{D}^{1}\left(B_{2}^{*}, B^{*}\right)=g_{D}^{2}\left(B_{2}^{*}, B^{*}\right)=g_{d}
\end{gathered}
$$

The above relation is confirmed by our detailed calculation.

For deriving the sum rules for the coupling constants we consider the correlator

$$
\begin{array}{r}
\int d^{4} x e^{-i k \cdot x}\left\langle\gamma(q)\left|T\left(J_{0,-, \frac{1}{2}}(0) J_{1,+, \frac{3}{2}}^{\dagger \alpha}(x)\right)\right| 0\right\rangle \\
=e_{q} e\left\{e_{\beta}^{*}\left(q_{t}^{\alpha} q_{t}^{\beta}-\frac{1}{3} q_{t}^{2} g_{t}^{\alpha \beta}\right) G_{B_{1} B}^{D}\left(\omega, \omega^{\prime}\right)+e_{t}^{* \alpha} G_{B_{1} B}^{S}\left(\omega, \omega^{\prime}\right)\right\}
\end{array} .
$$

The functions $G_{B_{1} B}^{D, S}\left(\omega, \omega^{\prime}\right)$ in (35) have the following double dispersion relation

$$
\frac{f_{-, \frac{1}{2}} f_{+, \frac{3}{2}} g_{D, S}\left(B_{1} B\right)}{\left(2 \bar{\Lambda}_{-, \frac{1}{2}}-\omega^{\prime}\right)\left(2 \bar{\Lambda}_{+, \frac{3}{2}}-\omega\right)}+\frac{c}{2 \bar{\Lambda}_{-, \frac{1}{2}}-\omega^{\prime}}+\frac{c^{\prime}}{2 \bar{\Lambda}_{+, \frac{3}{2}}-\omega},
$$


where $\bar{\Lambda}_{P, j_{\ell}}=m_{P, j_{\ell}}-m_{Q}$ and $f_{P, j_{\ell}}$ are constants defined as:

$$
\left\langle 0\left|J_{j, P, j_{\ell}}^{\alpha_{1} \cdots \alpha_{j}}(0)\right| j^{\prime}, P^{\prime}, j_{\ell}^{\prime}\right\rangle=f_{P j_{l}} \delta_{j j^{\prime}} \delta_{P P^{\prime}} \delta_{j \ell j_{\ell}} \eta^{\alpha_{1} \cdots \alpha_{j}} .
$$

Applying the same procedure as in section 2 we obtain

$$
\begin{array}{r}
G_{B_{1} B}^{D}\left(\omega, \omega^{\prime}\right)=-\frac{\sqrt{6}}{24} \int_{0}^{\infty} d t \int_{0}^{1} d u e^{i(1-u) \frac{\omega t}{2}} e^{i u \frac{\omega^{\prime} t}{2}} u\left\{-\frac{i t}{4} f \psi(u)\right. \\
\left.+\langle\bar{q} q\rangle\left[\chi \phi(u)+t^{2}\left(g_{1}(u)-g_{2}(u)\right)\right]\right\}+\cdots \\
G_{B_{1} B}^{S}\left(\omega, \omega^{\prime}\right)=-\frac{2}{3} q_{t}^{2} G_{B_{1} B}^{D}\left(\omega, \omega^{\prime}\right)=\frac{1}{6}\left(\omega-\omega^{\prime}\right)^{2} G_{B_{1} B}^{D}\left(\omega, \omega^{\prime}\right) .
\end{array}
$$

Finally we have:

$$
\begin{aligned}
& g_{d} f_{-, \frac{1}{2}} f_{+, \frac{3}{2}}=\frac{1}{8} e^{\frac{\Lambda_{-, \frac{1}{2}}+\Lambda}{T}+, \frac{3}{2}}\left\{f u_{0} \psi\left(u_{0}\right)+\frac{a}{2 \pi^{2}} u_{0}\left[\chi \phi\left(u_{0}\right) T f_{0}\left(\frac{\omega_{c}}{T}\right)-\frac{4}{T}\left(g_{1}\left(u_{0}\right)-g_{2}\left(u_{0}\right)\right)\right]\right\}, \\
& g_{s} f_{-, \frac{1}{2}} f_{+, \frac{3}{2}}=-\frac{1}{96} e^{\frac{\Lambda_{-, \frac{1}{2}}+\Lambda_{+, \frac{3}{2}}}{T}}\left\{f \frac{d^{2}(u \psi(u))}{d u^{2}} T^{2} f_{1}\left(\frac{\omega_{c}}{T}\right)+\frac{a}{2 \pi^{2}}\left[\chi \frac{d^{2}(u \phi(u))}{d u^{2}} T^{3} f_{2}\left(\frac{\omega_{c}}{T}\right)\right.\right. \\
& \left.\left.-4 \frac{d^{2}\left(\left(u g_{1}(u)-u g_{2}(u)\right)\right)}{d u^{2}} T f_{0}\left(\frac{\omega_{c}}{T}\right)\right]\right\}\left.\right|_{u=u_{0}}
\end{aligned}
$$

Here we have used integration by parts to absorb the factor $(q \cdot v)^{2}$, which leads to the second derivatives in (41). In this way we arrive at the simple form after double Borel transformation.

- $\left(0^{+}, 1^{+}\right) \rightarrow\left(0^{-}, 1^{-}\right)+\gamma$

There exists only one independent coupling constant, corresponding to S-wave decay. The decay amplitudes are:

$$
M\left(B_{1}^{\prime} \rightarrow B^{*} \gamma\right)=e_{q} e \epsilon^{\mu \sigma \alpha \beta} e_{\mu} v_{\sigma} \eta_{\alpha}^{\prime} \epsilon_{\beta}^{*} g_{S}\left(B_{1}^{\prime}, B^{*}\right),
$$

where $\eta_{\alpha}^{\prime}$ is the polarization vector of $B_{1}^{\prime}$.

$$
\begin{gathered}
M\left(B_{1}^{\prime} \rightarrow B \gamma\right)=e_{q} e e^{\alpha} \eta_{\alpha}^{\prime} g_{S}\left(B_{1}^{\prime}, B\right), \\
M\left(B_{0}^{\prime} \rightarrow B^{*} \gamma\right)=e_{q} e e_{t}^{\beta} \epsilon_{\beta} g_{S}\left(B_{0}^{\prime}, B^{*}\right) .
\end{gathered}
$$

The process $B_{0}^{\prime} \rightarrow B \gamma$ is forbidden due to parity and angular momentum conservation. Due to heavy quark symmetry, we have

$$
g_{S}\left(B_{1}^{\prime}, B^{*}\right)=g_{S}\left(B_{1}^{\prime}, B\right)=-g_{S}\left(B_{0}^{\prime}, B^{*}\right) \equiv g_{1} .
$$

We consider the correlator

$$
\int d^{4} x e^{-i k \cdot x}\left\langle\gamma(q)\left|T\left(J_{0,+, \frac{1}{2}}(0) J_{1,-, \frac{1}{2}}^{\dagger \beta}(x)\right)\right| 0\right\rangle=e_{q} e e^{\beta} G_{B_{0}^{\prime} B^{*}}\left(\omega, \omega^{\prime}\right),
$$


where

$$
\begin{gathered}
G_{B_{0}^{\prime} B^{*}}\left(\omega, \omega^{\prime}\right)=-\frac{1}{4}\langle\bar{q} q\rangle(q \cdot v) \int_{0}^{\infty} d t \int_{0}^{1} d u e^{i(1-u) \frac{\omega t}{2}} e^{i u \frac{\omega^{\prime} t}{2}}\left\{\chi \phi(u)+t^{2} g_{1}(u)\right\}+\cdots . \\
g_{1} f_{-, \frac{1}{2}} f_{+, \frac{1}{2}}=\left.\frac{a}{16 \pi^{2}} e^{\frac{\Lambda_{-, \frac{1}{2}}+\Lambda}{T}}\left\{\chi \frac{d \phi(u)}{d u} T^{2} f_{1}\left(\frac{\omega_{c}}{T}\right)-4 \frac{d g_{1}(u)}{d u}\right\}\right|_{u=u_{0}} .
\end{gathered}
$$

- $\left(1^{+}, 2^{+}\right) \rightarrow\left(0^{+}, 1^{+}\right)+\gamma$

There exists only one independent coupling constant, corresponding to P-wave decay. The decay amplitudes are:

$$
\begin{gathered}
M\left(B_{1} \rightarrow B_{1}^{\prime} \gamma\right)=e_{q} e \eta_{\alpha} \eta_{\beta}^{\prime *} e_{\mu}^{*}\left\{\left[\left(q_{t}^{\alpha} g_{t}^{\beta \mu}-\frac{1}{3} q_{t}^{\mu} g_{t}^{\alpha \beta}\right)+(\alpha \leftrightarrow \beta)\right] g_{P}^{1}\left(B_{1}, B_{1}^{\prime}\right)\right. \\
\left.+\left(q_{t}^{\alpha} g_{t}^{\beta \mu}-q_{t}^{\beta} g_{t}^{\alpha \mu}\right) g_{P}^{2}\left(B_{1}, B_{1}^{\prime}\right)\right\} \\
M\left(B_{1} \rightarrow B_{0}^{\prime} \gamma\right)=e_{q} e \epsilon_{\mu \sigma \nu \alpha} \eta^{\alpha} e^{* \mu} v^{\sigma} q^{\nu} g_{P}\left(B_{1}, B_{0}^{\prime}\right), \\
M\left(B_{2}^{*} \rightarrow B_{0}^{\prime} \gamma\right)=e_{q} e e^{* \mu} \eta_{\alpha_{1} \alpha_{2}}\left[\left(q_{t}^{\alpha_{1}} g_{t}^{\alpha_{2} \mu}-\frac{1}{3} q_{t}^{\mu} g_{t}^{\alpha_{1} \alpha_{2}}\right)+\left(\alpha_{1} \leftrightarrow \alpha_{2}\right)\right] g_{P}\left(B_{2}^{*}, B_{0}^{\prime}\right), \\
M\left(B_{2}^{*} \rightarrow B_{1}^{\prime} \gamma\right)=e_{q} e \epsilon_{\mu \sigma \rho \beta} \eta^{\prime \beta} e^{* \mu} \eta_{\alpha_{1} \alpha_{2}}\left[\left(q_{t}^{\alpha_{1}} g_{t}^{\alpha_{2} \rho}-\frac{1}{3} q_{t}^{\rho} g_{t}^{\alpha_{1} \alpha_{2}}\right)+\left(\alpha_{1} \leftrightarrow \alpha_{2}\right)\right] g_{P}\left(B_{2}^{*}, B_{1}^{\prime}\right) .
\end{gathered}
$$

Due to heavy quark symmetry we have

$$
\frac{\sqrt{6}}{3} g_{P}^{1}\left(B_{1}, B_{1}^{\prime}\right)=\sqrt{6} g_{P}^{2}\left(B_{1}, B_{1}^{\prime}\right)=\sqrt{6} g_{P}\left(B_{1}, B_{0}^{\prime}\right)=g_{P}\left(B_{2}^{*}, B_{0}^{\prime}\right)=g_{P}\left(B_{2}^{*}, B_{1}^{\prime}\right) \equiv g_{2} .
$$

We consider the correlator

$$
\begin{array}{r}
\int d^{4} x e^{-i k \cdot x}\left\langle\pi(q)\left|T\left(J_{0,+, \frac{1}{2}}^{\alpha}(0) J_{2,+, \frac{3}{2}}^{\dagger \alpha_{1} \alpha_{2}}(x)\right)\right| 0\right\rangle= \\
e_{q} e e^{\mu}\left[\left(q_{t}^{\alpha_{1}} g_{t}^{\alpha_{2} \mu}-\frac{1}{3} q_{t}^{\mu} g_{t}^{\alpha_{1} \alpha_{2}}\right)+\left(\alpha_{1} \leftrightarrow \alpha_{2}\right)\right] G_{B_{2}^{*} B_{0}^{\prime}}\left(\omega, \omega^{\prime}\right)
\end{array}
$$

where

$$
\begin{gathered}
G_{B_{2}^{*} B_{0}^{\prime}}\left(\omega, \omega^{\prime}\right)=-\frac{1}{8}\langle\bar{q} q\rangle(q \cdot v) \int_{0}^{\infty} d t \int_{0}^{1} d u e^{i(1-u) \frac{\omega t}{2}} e^{i u \frac{\omega^{\prime} t}{2}} u\left\{\chi \phi(u)+t^{2} g_{1}(u)\right\}+\cdots \\
g_{2} f_{+, \frac{1}{2}} f_{+, \frac{3}{2}}=\left.\frac{a}{32 \pi^{2}} e^{\frac{\Lambda_{+, \frac{1}{2}}+\Lambda_{+}, \frac{3}{2}}{T}}\left\{\chi \frac{d(u \phi(u))}{d u} T^{2} f_{1}\left(\frac{\omega_{c}}{T}\right)-4 \frac{d\left(u g_{1}(u)\right)}{d u}\right\}\right|_{u=u_{0}}
\end{gathered}
$$

- $B_{1}^{\prime} \rightarrow B_{0}^{\prime} \gamma$

$$
M\left(B_{1}^{\prime} \rightarrow B_{0}^{\prime} \gamma\right)=e_{q} e e^{\alpha \mu \nu \sigma} \eta_{\alpha}^{\prime} e_{\mu}^{*} q_{\nu} v_{\sigma} g_{3}
$$


In order to derive $g_{3}$, we consider the correlator

$$
\int d^{4} x e^{-i k \cdot x}\left\langle\gamma(q)\left|T\left(J_{0,+, \frac{1}{2}}(0) J_{1,+, \frac{1}{2}}^{\dagger \alpha}(x)\right)\right| 0\right\rangle=e_{q} e \epsilon^{\alpha \mu \nu \sigma} e_{\mu} q_{\nu} v_{\sigma} G_{B_{1}^{\prime} B_{0}^{\prime}}\left(\omega, \omega^{\prime}\right),
$$

where

$$
\begin{gathered}
G_{B_{1}^{\prime} B_{0}^{\prime}}\left(\omega, \omega^{\prime}\right)=\frac{i}{4} \int_{0}^{\infty} d t \int_{0}^{1} d u e^{i(1-u) \frac{\omega t}{2}} e^{i u \frac{\omega^{\prime} t}{2}}\left\{\frac{i t}{4} f \psi(u)+\langle\bar{q} q\rangle[\chi \phi(u)\right. \\
\left.\left.+t^{2}\left(g_{1}(u)-g_{2}(u)\right)\right]\right\}+\cdots \\
g_{3} f_{+, \frac{1}{2}}^{2}=-\frac{1}{4} e^{\frac{2 \Lambda_{+}, \frac{1}{2}}{T}}\left\{-f \psi\left(u_{0}\right)+\frac{a}{2 \pi^{2}}\left[\chi \phi\left(u_{0}\right) T f_{0}\left(\frac{\omega_{c}}{T}\right)-\frac{4}{T}\left(g_{1}\left(u_{0}\right)-g_{2}\left(u_{0}\right)\right)\right]\right\} .
\end{gathered}
$$

- $B^{*} \rightarrow B \gamma$

$$
M\left(B^{*} \rightarrow B \gamma\right)=e_{q} e \epsilon^{\alpha \mu \nu \sigma} \epsilon_{\alpha} e_{\mu}^{*} q_{\nu} v_{\sigma} g_{4} .
$$

In order to derive $g_{4}$, we consider the correlator

$$
\int d^{4} x e^{-i k \cdot x}\left\langle\gamma(q)\left|T\left(J_{0,-, \frac{1}{2}}(0) J_{1,-, \frac{1}{2}}^{\dagger \alpha}(x)\right)\right| 0\right\rangle=e_{q} e \epsilon^{\alpha \mu \nu \sigma} e_{\mu} q_{\nu} v_{\sigma} G_{B^{*} B}\left(\omega, \omega^{\prime}\right),
$$

where

$$
\begin{aligned}
G_{B^{*} B}\left(\omega, \omega^{\prime}\right)=\frac{i}{4} \int_{0}^{\infty} d t \int_{0}^{1} d u e^{i(1-u) \frac{\omega t}{2}} e^{i u \frac{\omega^{\prime} t}{2}}\left\{-\frac{i t}{4} f \psi(u)+\langle\bar{q} q\rangle[\chi \phi(u)\right. \\
\left.\left.+t^{2}\left(g_{1}(u)-g_{2}(u)\right)\right]\right\}+\cdots .
\end{aligned}
$$

Note this coupling was calculated in [3] using LCQSR. But there the contribution from the photon wave function $\psi(u)$ has not been taken into account.

$$
g_{4} f_{-, \frac{1}{2}}^{2}=-\frac{1}{4} e^{\frac{2 \Lambda}{T-\frac{1}{2}}}\left\{f \psi\left(u_{0}\right)+\frac{a}{2 \pi^{2}}\left[\chi \phi\left(u_{0}\right) T f_{0}\left(\frac{\omega_{c}}{T}\right)-\frac{4}{T}\left(g_{1}\left(u_{0}\right)-g_{2}\left(u_{0}\right)\right)\right]\right\} .
$$

- $B_{2}^{*} \rightarrow B_{1} \gamma$

$$
M\left(B_{2}^{*} \rightarrow B_{1} \gamma\right)=e_{q} e \epsilon^{\alpha \mu \nu \sigma} e_{\mu} q_{\nu} v_{\sigma} \eta_{\beta}^{*} \eta_{\alpha_{1} \alpha_{2}}\left(g_{t}^{\alpha_{1} \rho} q_{t}^{\alpha_{2}}+g_{t}^{\alpha_{2} \rho} q_{t}^{\alpha_{1}}-\frac{2}{3} g_{t}^{\alpha_{1} \alpha_{2}} q_{t}^{\rho}\right)\left(2 q_{t}^{\beta} g_{t}^{\rho \alpha}+g_{t}^{\alpha \beta} q_{t}^{\rho}\right) g_{5}
$$

In order to derive $g_{5}$, we consider the correlator

$$
\begin{array}{r}
\int d^{4} x e^{-i k \cdot x}\left\langle\gamma(q)\left|T\left(J_{1,+, \frac{3}{2}}^{\beta}(0) J_{2,+, \frac{3}{2}}^{\dagger \alpha_{1} \alpha_{2}}(x)\right)\right| 0\right\rangle= \\
e_{q} e \epsilon^{\alpha \mu \nu \sigma} e_{\mu} q_{\nu} v_{\sigma}\left(g_{t}^{\alpha_{1} \rho} q_{t}^{\alpha_{2}}+g_{t}^{\alpha_{2} \rho} q_{t}^{\alpha_{1}}-\frac{2}{3} g_{t}^{\alpha_{1} \alpha_{2}} q_{t}^{\rho}\right)\left(2 q_{t}^{\beta} g_{t}^{\rho \alpha}+g_{t}^{\alpha \beta} q_{t}^{\rho}\right) G_{B_{2}^{*} B_{1}}\left(\omega, \omega^{\prime}\right)
\end{array}
$$


where

$$
\begin{gathered}
G_{B_{2}^{*} B_{1}}\left(\omega, \omega^{\prime}\right)=-i \frac{\sqrt{6}}{16} \int_{0}^{\infty} d t \int_{0}^{1} d u e^{i(1-u) \frac{\omega t}{2}} e^{i u \frac{\omega^{\prime} t}{2}} u(1-u)\left\{-\frac{i t}{4} f \psi(u)\right. \\
\left.+\langle\bar{q} q\rangle\left[\chi \phi(u)+t^{2}\left(g_{1}(u)-g_{2}(u)\right)\right]\right\}+\cdots
\end{gathered}
$$

\section{Determination of the parameters}

The leading photon wave functions receive only small corrections from the higher conformal spins [9] so they do not deviate much from the asymptotic form. We shall use [4]

$$
\begin{gathered}
\phi(u)=6 u \bar{u}, \\
\psi(u)=1, \\
g_{1}(u)=-\frac{1}{8} \bar{u}(3-u), \\
g_{2}(u)=-\frac{1}{4} \bar{u}^{2} .
\end{gathered}
$$

with $f=0.028 \mathrm{GeV}^{2}$ and $\chi=-4.4 \mathrm{GeV}^{2}$ 13 at the scale $\mu=1 \mathrm{GeV}$. Using this value of $\chi$, the octet, decuplet and heavy baryon magnetic moments have been calculated to a good accuracy [14, 15, 16].

We need the mass parameters $\bar{\Lambda}$ 's and the coupling constants $f$ 's of the corresponding interpolating currents in the leading order of $\alpha_{s}$ as input. The results are [11, 8]

$$
\begin{aligned}
& \bar{\Lambda}_{\Lambda}=0.8 \mathrm{GeV} \quad f_{\Lambda}=(0.018 \pm 0.002) \mathrm{GeV}^{3}, \\
& \bar{\Lambda}_{\Sigma}=1.0 \mathrm{GeV} \quad f_{\Sigma}=(0.04 \pm 0.004) \mathrm{GeV}^{3} . \\
& \bar{\Lambda}_{+, 3 / 2}=0.82 \mathrm{GeV} \quad f_{+, 3 / 2}=0.19 \pm 0.03 \mathrm{GeV}^{5 / 2} \\
& \bar{\Lambda}_{+, 1 / 2}=1.1 \mathrm{GeV} \quad f_{+, 1 / 2}=0.40 \pm 0.06 \mathrm{GeV}^{3 / 2} \text {, } \\
& \bar{\Lambda}_{-, 1 / 2}=0.5 \mathrm{GeV} \quad f_{-, 1 / 2}=0.25 \mathrm{GeV}^{3 / 2} .
\end{aligned}
$$

We choose to work at the symmetric point $T_{1}=T_{2}=2 T$, i.e., $u_{0}=\frac{1}{2}$. Such a choice is very reasonable for the symmetric sum rules (25), (60), (64) and (68) since $\Sigma_{c}^{*}$ and $\Sigma_{c}$, and the three meson doublets are degenerate in the leading order of HQET. Moreover, the mass difference between $\Sigma_{c}^{*}$ and $\Lambda_{c}$ is only about $0.2 \mathrm{GeV}$. The $\left(0^{+}, 1^{+}\right)$doublet lies only slightly below $\left(1^{+}, 2^{+}\right)$doublet. Due to the large values of $T_{1}, T_{2}$ used below, the choice of $T_{1}=T_{2}$ is also reasonable for sum rules (23) and (56).

Note the choice $T_{1}=T_{2}$ is not unique for the asymmetric sum rules (40), (41) and (48) since the initial and final mesons have different masses. But the choice $T_{1}=T_{2}$ will enable 
the clean subtraction of the continuum contribution, which is cruicial for the numerical analysis of the sum rules. In our case the sum rules are stable with reasonable variations of the Borel parameter $T_{1}$ and $T_{2}$. Such a choice does not alter significantly the numerical

results. Based on these considerations we adopt $u_{0}=\frac{1}{2}$ for for the sum rules (40), (41) and (48) too.

\section{Numerical results and discussion}

\subsection{Numerical analysis of the baryon sum rules}

We now turn to the numerical evaluation of the sum rules for the coupling constants. Since the spectral density of the sum rule (23)-(25) $\rho(s)$ is either proptional to $s^{2}$ or $s^{3}$, the continuum has to be subtracted carefully. We use the value of the continuum threshold $\omega_{c}$ determined from the corresponding mass sum rule at the leading order of $\alpha_{s}$ and $1 / m_{Q}$ 11.

The lower limit of $T$ is determined by the requirement that the terms of higher twists in the operator expansion is reasonably smaller than the leading twist, say $\leq 1 / 3$ of the latter. This leads to $T>1.3 \mathrm{GeV}$ for the sum rules (23)-(25). In fact the twist-four terms contribute only a few percent to the sum rules. The upper limit of $T$ is constrained by the requirement that the continuum contribution is less than $50 \%$. This corresponds to $T<2.2 \mathrm{GeV}$.

The variation of $\eta_{1,3}$ with the Borel parameter $T$ and $\omega_{c}$ is presented in FIG. 1 and FIG. 2. The curves correspond to $\omega_{c}=2.4,2.5,2.6 \mathrm{GeV}$ from bottom to top respectively. Stability develops for the sum rules (23) and (25) in the region $1.3 \mathrm{GeV}<T<2.2 \mathrm{GeV}$, we get:

$$
\begin{aligned}
& \eta_{1} f_{\Sigma} f_{\Lambda}=(7.0 \pm 0.9) \times 10^{-4} \mathrm{GeV}^{5} \\
& \eta_{3} f_{\Sigma^{*}} f_{\Sigma}=(3.9 \pm 0.5) \times 10^{-4} \mathrm{GeV}^{5}
\end{aligned}
$$

where the errors refers to the variations with $T$ and $\omega_{c}$ in this region. And the central value corresponds to $T=1.6 \mathrm{GeV}$ and $\omega_{c}=2.5 \mathrm{GeV}$.

Combining $(73)$ we arrive at

$$
\begin{aligned}
& \eta_{1}=(1.0 \pm 0.2) \mathrm{GeV}^{-1} \\
& \eta_{3}=(0.24 \pm 0.05) \mathrm{GeV}^{-1}
\end{aligned}
$$

\subsection{Numerical analysis of the meson sum rules}

We now turn to the numerical evaluation of the sum rules for the coupling constants. The lower limit of $T$ is determined by the requirement that the terms of higher twists in the operator expansion is less than one third of the whole sum rule. This leads to $T>1.0$ $\mathrm{GeV}$ for the sum rules (40), (41), (48), (56), (60), (64) and (68). In fact the twist-four terms contribute only a few percent to the sum rules for such $T$ values. The upper limit of $T$ is constrained by the requirement that the continuum contribution is less than $30 \%$. This corresponds to $T<2.5 \mathrm{GeV}$. With the values of photon wave functions at $u_{0}=\frac{1}{2}$ we 
obtain the left hand side of these sum rules as functions of $T$. The continuum threshold is $\omega_{c}=3.0 \pm 0.2 \mathrm{GeV}$ except $\omega_{c}=2.4 \pm 0.2 \mathrm{GeV}$ for the sum rule (64). Stability develops for the sum rules in the region $1.0 \mathrm{GeV}<T<2.5 \mathrm{GeV}$. The results are shown in FIG. 3-9. Numerically we have:

$$
\begin{aligned}
& g_{d} f_{-, \frac{1}{2}} f_{+, \frac{3}{2}}=-(3.0 \pm 0.2) \times 10^{-2} \quad \mathrm{GeV}^{2} \\
& g_{s} f_{-, \frac{1}{2}} f_{+, \frac{3}{2}}=-(1.9 \pm 0.2) \times 10^{-2} \mathrm{GeV}^{4} \\
& g_{1} f_{-, \frac{1}{2}} f_{+, \frac{1}{2}}=-(1.5 \pm 0.5) \times 10^{-2} \mathrm{GeV}^{3} \\
& g_{2} f_{+, \frac{1}{2}} f_{+, \frac{3}{2}}=-(5.5 \pm 0.4) \times 10^{-2} \quad \mathrm{GeV}^{3} \\
& g_{3} f_{+, \frac{1}{2}}^{2}=(0.28 \pm 0.04) \quad \mathrm{GeV}^{2} \\
& g_{4} f_{-, \frac{1}{2}}^{2}=(8.9 \pm 0.5) \times 10^{-2} \quad \mathrm{GeV}^{2} \\
& g_{5} f_{+, \frac{3}{2}}^{2}=-(2.3 \pm 0.3) \times 10^{-2} \mathrm{GeV}^{2}
\end{aligned}
$$

where the errors refer to the variations with $T$ in this region and the uncertainty in $\omega_{c}$. And the central value corresponds to $T=1.5 \mathrm{GeV}$ and $\omega_{c}=3.0 \pm 0.2 \mathrm{GeV}$ except that we use $\omega_{c}=2.4 \pm 0.2 \mathrm{GeV}$ for the sum rule (64).

With the central values of f's in (74) we get the absolute value of the coupling constants:

$$
\begin{aligned}
& g_{d}=-(0.63 \pm 0.10) \quad \mathrm{GeV}^{-2} \\
& g_{s}=-(0.40 \pm 0.05) \\
& g_{1}=-(0.20 \pm 0.06) \\
& g_{2}=-(0.72 \pm 0.07) \quad \mathrm{GeV}^{-1} \\
& g_{3}=(1.8 \pm 0.3) \quad \mathrm{GeV}^{-1} \\
& g_{4}=(1.4 \pm 0.2) \quad \mathrm{GeV}^{-1} \\
& g_{5}=-(0.64 \pm 0.08) \quad \mathrm{GeV}^{-3}
\end{aligned}
$$

Note we have only considered the uncertainty due to the variations of the Borel parameter and the continuum threshold in the above expressions. There are other sources of uncertainty. The input parameters $\chi$ and $f$ are associated with the photon distribution amplitude. Especially the value of $\chi$ has been estimated with QCD sum rules [13] and with the octet baryon magnetic moments as inputs using the external field method [15]. Both approaches yield consistent results $\chi \approx-4.4 \mathrm{GeV}$. With this value the octet, decuplet and heavy baryon magnetic moments derived using the external field method are in good agreement with the experimental data. So we expect its accuracy is better than $30 \%$. The value of $f$ has been estimated with the vector meson dominance model also with an accuracy of $30 \%$ [2].

The light cone sum rules for the coupling constants $g_{i}$ and the mass sum rules for the heavy hadrons in HQET both receive large perturbative QCD corrections. But their ratio does not depend on radiative corrections strongly because of large cancellation [17]. In the present case, the uncertainty of the coupling constants $g_{i}$ due to radiative corrections is expected to around $10 \%$ while the couplings $f_{\Lambda}$ etc are affected significantly.

Another possible source of error is the truncation of the light cone expansion at twist four. We take the sum rules for $\eta_{1}$ for example. At $T=1.5 \mathrm{GeV}$, the twist-four term 
involved with $g_{1}, g_{2}$ is only $-2.5 \%$ of the leading twist term after making double Borel transformation to (20). Even after the subtraction of the continuum and excited states contribution the twist-four term is only $-15 \%$ of the twist-two one in (23). So the light cone expansion converges quickly. We expect the contribution of higher twist distribution amplitudes to be small.

We have calculated the coupling constant in the leading order of HQET. The $1 / m_{Q}$ correction is sizable for the charm system. But for the bottom system the $1 / m_{Q}$ correction is typically around $5 \% \sim 10 \%$ for the pionic coupling constants [8]. We expect the $1 / m_{Q}$ correction to the electromagnetic coupling constants is of the same order. The inherent uncertainty of the method of QCD sum rules is not included, which is typically about $10 \%$.

\subsection{Decay widths of heavy hadrons}

With these coupling constants we can calculate the decay widths of heavy hadrons.

The decay width formulas in the leading order of HQET are

$$
\begin{aligned}
& \Gamma\left(\Sigma_{b} \rightarrow \Lambda_{b} \gamma\right)=4 \eta_{1}^{2} \alpha|\vec{q}|^{3} \\
& \Gamma\left(\Sigma_{b}^{*} \rightarrow \Lambda_{b} \gamma\right)=\eta_{2}^{2} \alpha|\vec{q}|^{3} \frac{3 m_{i}^{2}+m_{f}^{2}}{3 m_{i}^{2}} \\
& \Gamma\left(\Sigma_{b}^{*} \rightarrow \Sigma_{b} \gamma\right)=\eta_{3}^{2} \alpha|\vec{q}|^{3} \frac{3 m_{i}^{2}+m_{f}^{2}}{3 m_{i}^{2}} \\
& \Gamma\left(B_{1} \rightarrow B^{*} \gamma\right)=\frac{2}{3} e_{q}^{2} \alpha\left(\frac{14}{9} g_{d}^{2}|\vec{q}|^{5}+g_{s}^{2}|\vec{q}|\right) \\
& \Gamma\left(B_{1} \rightarrow B \gamma\right)=\frac{4 \alpha}{3} e_{q}^{2} \alpha\left(\frac{1}{18} g_{d}^{2}|\vec{q}|^{5}+g_{s}^{2}|\vec{q}|\right) \\
& \Gamma\left(B_{2}^{*} \rightarrow B \gamma\right)=\frac{2}{5} e_{q}^{2} \alpha g_{d}^{2}|\vec{q}|^{5} \\
& \Gamma\left(B_{2}^{*} \rightarrow B^{*} \gamma\right)=e_{q}^{2} \alpha\left(\frac{64}{45} g_{d}^{2}|\vec{q}|^{5}+4 g_{s}^{2}|\vec{q}|\right) \\
& \Gamma\left(B_{1}^{\prime} \rightarrow B^{*} \gamma\right)=e_{q}^{2} \alpha g_{1}^{2}|\vec{q}| \\
& \Gamma\left(B_{1}^{\prime} \rightarrow B \gamma\right)=\frac{1}{2} e_{q}^{2} \alpha g_{1}^{2}|\vec{q}| \\
& \Gamma\left(B_{0}^{\prime} \rightarrow B^{*} \gamma\right)=\frac{3}{2} e_{q}^{2} \alpha g_{1}^{2}|\vec{q}| \\
& \Gamma\left(B^{*} \rightarrow B \gamma\right)=\frac{1}{3} e_{q}^{2} \alpha g_{4}^{2}|\vec{q}|^{3}
\end{aligned}
$$

where $|\vec{q}|=\frac{m_{i}^{2}-m_{f}^{2}}{2 m_{i}}, m_{i}, m_{f}$ is the parent and decay heavy hadron mass.

We apply the leading order formulas obtained above to the excited states of bottomed hadrons using the central values of the coupling constants in the previous section.

$$
\begin{aligned}
& \Gamma\left(\Sigma_{b} \rightarrow \Lambda_{b} \gamma\right)=131 \times\left(\frac{|\vec{q}|}{165 \mathrm{MeV}}\right)^{3} \mathrm{keV}, \\
& \Gamma\left(\Sigma_{b}^{* 0} \rightarrow \Lambda_{b} \gamma\right)=313 \times\left(\frac{|\vec{q}|}{224 \mathrm{MeV}}\right)^{3} \mathrm{keV},
\end{aligned}
$$




$$
\begin{aligned}
& \Gamma\left(\Sigma_{b}^{*+} \rightarrow \Sigma_{b}^{+} \gamma\right)=2.2 \times\left(\frac{|\vec{q}|}{63.4 \mathrm{MeV}}\right)^{3} \mathrm{keV} \\
& \Gamma\left(\Sigma_{b}^{* 0} \rightarrow \Sigma_{b}^{0} \gamma\right)=0.14 \times\left(\frac{|\vec{q}|}{63.4 \mathrm{MeV}}\right)^{3} \mathrm{keV} \\
& \Gamma\left(\Sigma_{b}^{*-} \rightarrow \Sigma_{b}^{-} \gamma\right)=0.56 \times\left(\frac{|\vec{q}|}{63.4 \mathrm{MeV}}\right)^{3} \mathrm{keV}, \\
& \Gamma\left(B^{* 0} \rightarrow B^{0} \gamma\right)=1.4 \times\left(\frac{|\vec{q}|}{137 \mathrm{MeV}}\right) \mathrm{keV}, \\
& \Gamma\left(B^{*+} \rightarrow B^{+} \gamma\right)=5.5 \times\left(\frac{|\vec{q}|}{137 \mathrm{MeV}}\right) \mathrm{keV}, \\
& \Gamma\left(B_{1}^{0} \rightarrow B^{0} \gamma\right)=84.4 \times\left(\frac{|\vec{q}|}{490 \mathrm{MeV}}\right) \mathrm{keV} \\
& \Gamma\left(B_{1}^{+} \rightarrow B^{+} \gamma\right)=338 \times\left(\frac{|\vec{q}|}{490 \mathrm{MeV}}\right) \mathrm{keV}, \\
& \Gamma\left(B_{1}^{0} \rightarrow B^{* 0} \gamma\right)=42.2 \times\left(\frac{|\vec{q}|}{377 \mathrm{MeV}}\right) \mathrm{keV}, \\
& \Gamma\left(B_{1}^{+} \rightarrow B^{*+} \gamma\right)=169 \times\left(\frac{|\vec{q}|}{377 \mathrm{MeV}}\right) \mathrm{keV}, \\
& \Gamma\left(B_{2}^{* 0} \rightarrow B^{0} \gamma\right)=5.8 \times\left(\frac{|\vec{q}|}{537 \mathrm{MeV}}\right) \mathrm{keV}, \\
& \Gamma\left(B_{2}^{*+} \rightarrow B^{+} \gamma\right)=23 \times\left(\frac{|\vec{q}|}{537 \mathrm{MeV}}\right) \mathrm{keV}, \\
& \Gamma\left(B_{2}^{* 0} \rightarrow B^{* 0} \gamma\right)=211 \times\left(\frac{|\vec{q}|}{408 \mathrm{MeV}}\right) \mathrm{keV}, \\
& \Gamma\left(B_{2}^{*+} \rightarrow B^{*+} \gamma\right)=844 \times\left(\frac{|\vec{q}|}{408 \mathrm{MeV}}\right) \mathrm{keV} .
\end{aligned}
$$

The uncertainty of the decay width is typically about $30 \%$.

We do not present numerical results for the radiative decay widths for the charmed hadrons since $1 / m_{Q}$ corrections are sizable for the charm system while such corrections are only a few percent of the leading order term for the bottom system [8].

In summary we have calculated the coupling constants of photons with the heavy baryons and the lowest three heavy meson doublets using the light cone QCD sum rules with the photon wave functions in the leading order of HQET. We hope these calculations will be tested in the future experiments.

Acknowledgments: S.-L. Zhu was supported by the Postdoctoral Science Foundation of China and the Natural Science Foundation of China. Y.D. was supported by the Natural Science Foundation of China. 


\section{Figure Captions}

FIG. 1. Dependence of $f_{\Sigma} f_{\Lambda} \eta_{1}$ on the Borel parameter $T$ for different values of the continuum threshold $\omega_{c}$. From top to bottom the curves correspond to $\omega_{c}=2.6,2.5,2.4 \mathrm{GeV}$.

FIG. 2. Dependence of $f_{\Sigma^{*}} f_{\Sigma} \eta_{3}$ on $T$ with $\omega_{c}=2.6,2.5,2.4 \mathrm{GeV}$.

FIG. 3. Dependence of $g_{d} f_{-, \frac{1}{2}} f_{+, \frac{3}{2}}$ on $T$ with $\omega_{c}=3.2,3.0,2.8 \mathrm{GeV}$.

FIG. 4. Dependence of $g_{s} f_{-, \frac{1}{2}} f_{+, \frac{3}{2}}$ on $T$ with $\omega_{c}=3.2,3.0,2.8 \mathrm{GeV}$.

FIG. 5. Dependence of $g_{1} f_{-, \frac{1}{2}} f_{+, \frac{1}{2}}$ on $T$.

FIG. 6. Dependence of $g_{2} f_{+, \frac{1}{2}} f_{+, \frac{3}{2}}$ on $T$ with $\omega_{c}=3.2,3.0,2.8 \mathrm{GeV}$.

FIG. 7. Dependence of $g_{3} f_{+, \frac{1}{2}}^{2}$ on $T$ with $\omega_{c}=3.2,3.0,2.8 \mathrm{GeV}$.

FIG. 8. Dependence of $g_{4} f_{-, \frac{1}{2}}^{2}$ on $T$ with $\omega_{c}=2.6,2.4,2.2 \mathrm{GeV}$.

FIG. 9. Dependence of $g_{5} f_{+, \frac{3}{2}}^{2}$ on $T$ with $\omega_{c}=3.2,3.0,2.8 \mathrm{GeV}$.

\section{References}

[1] B. Grinstein, Nucl. Phys. B339, 253 (1990);

E. Eichten and B. Hill, Phys. Lett. B234, 511 (1990);

A. F. Falk, H. Georgi, B. Grinstein and M. B. Wise, Nucl. Phys. B343, 1 (1990);

F. Hussain, J. G. Körner, K. Schilcher, G. Thompson and Y. L. Wu, Phys. Lett. B249, 295 (1990);

H. Georgi, Phys. Lett. B240, 447 (1990);

J. G. Körner and G. Thompson, Phys. Lett. B264, 185 (1991).

[2] G. Eilam, I. Halperin and R. R. Mendel, Phys. Lett. B 361, 137 (1995).

[3] T. M. Aliev, D. A. Demir, E. Iltan and N. K. Pak, Phys. Rev. D 54, 857 (1996).

[4] A. Ali and V. M. Braun, Phys. Lett. B 359, 223 (1995).

[5] A. Khodjamirian, G. Stoll and D. Wyler, Phys. Lett. B 358, 129 (1995).

[6] P. Ball and V. M. Braun, hep-ph/9805422.

[7] M.A. Shifman, A.I. Vainshtein and V.I. Zakharov, Nucl. Phys. B 174, 385, 448, 519 (1979).

[8] Yuan-Ben Dai and Shi-Lin Zhu, Phys. Rev. D 58, 074009 (1998); Euro. Phys. J. C 6, 307 (1999); Shi-Lin Zhu and Yuan-Ben Dai, Phys. Lett. B 429, 72 (1998). 
[9] I. I. Balitsky, V. M. Braun and A. V. Kolesnichenko, Nucl. Phys. B 312, 509 (1989); V. M. Braun and I. E. Filyanov, Z. Phys. C 44, 157(1989);

V. M. Braun and I. E. Filyanov, Z. Phys. C 48, 239(1990);

V. M. Belyaev, V. M. Braun, A. Khodjamirian and R. Rückl, Phys. Rev. D 51, 6177(1995).

[10] Shi-Lin Zhu, W-Y. P. Hwang and Ze-sen Yang, Phys. Lett. B 420, 8 (1998); Mod. Phys. Lett. A 12, 3027 (1997).

[11] A. G. Grozin and O. I. Yakovlev, Phys. Lett. B285, 254 (1992);

S. Groote, J. G. Korner, and O. I. Yakovlev, Phys. Rev. D54, 3447 (1996); D55, 3016 (1997).

[12] Y. B. Dai, C. S. Huang, M. Q. Huang and C. Liu, Phys. Lett. B390, 350 (1997).

[13] V. M. Belyaev and Y. I. Kogan, Yad. Fiz. 40, 1035 (1984);

I. I. Balitsky and A. V. Kolesnichenko, Yad. Fiz. 41, 282 (1985).

[14] B. L. Ioffe and A. V. Smilga, Nucl. Phys. B 232, 109 (1984); I.I.Balitsky and A. V. Yung, Phys. Lett. B 129, 328 (1983).

[15] C. B. Chiu, J. Pasupathy and S. J. Wilson, Phys. Rev. D 33, 1961 (1986).

[16] Shi-Lin Zhu, W-Y. P. Hwang and Ze-sen Yang, Phys. Rev. D 57, 1527 (1998); Phys. Rev. D56, 7273 (1997).

[17] M. Neubert, Phys. Rep. 245, 259 (1994). 


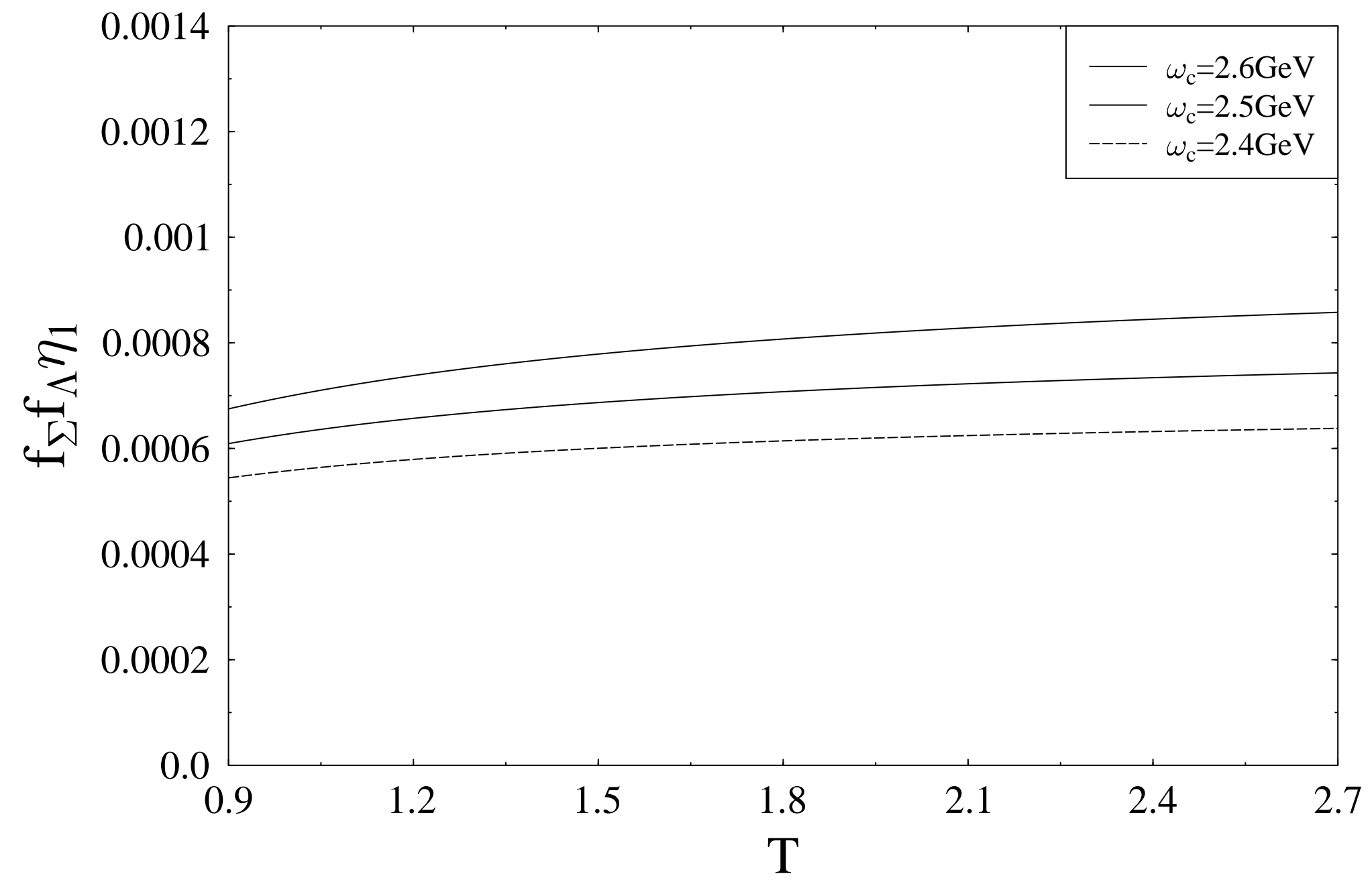




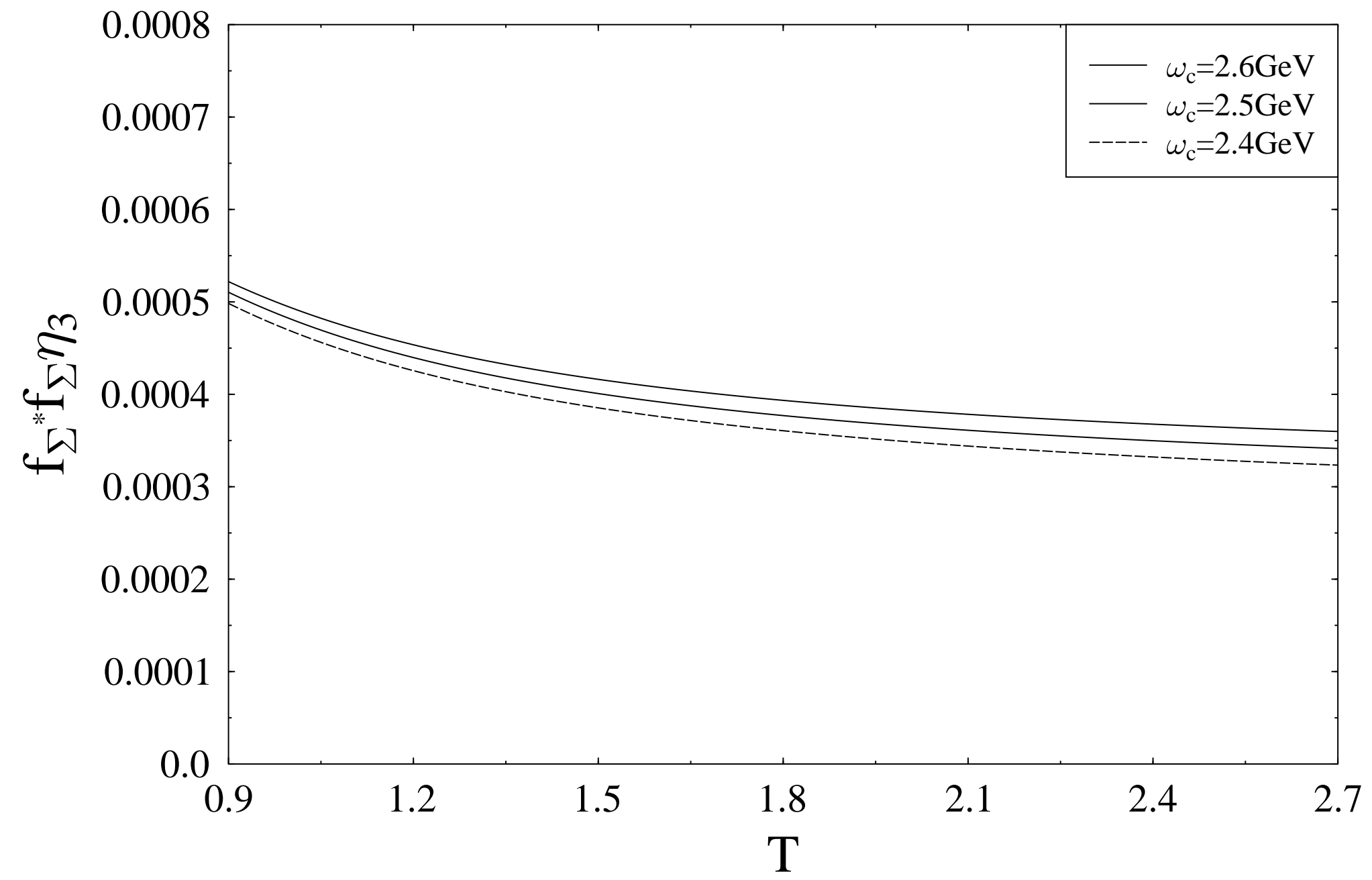




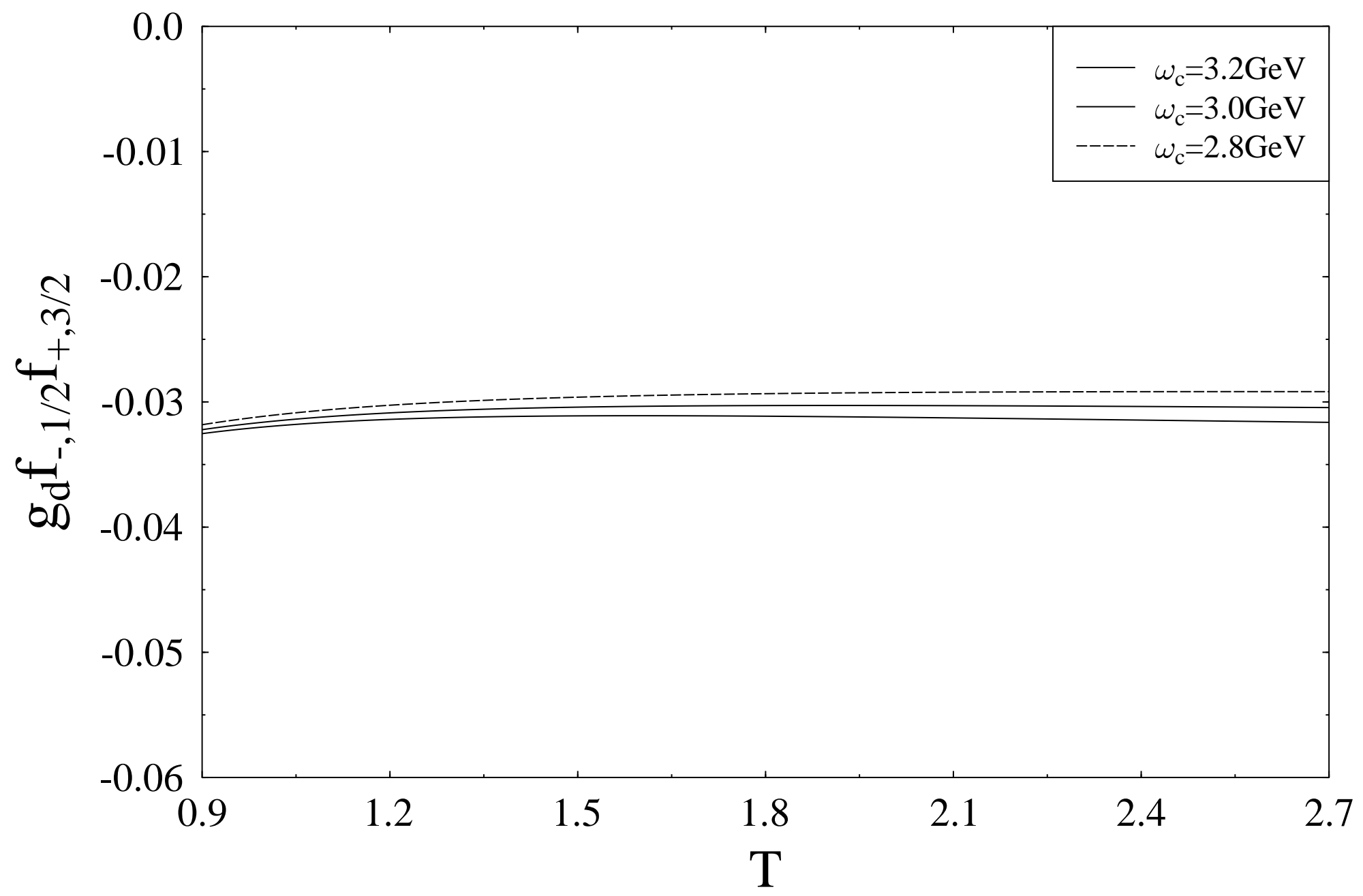




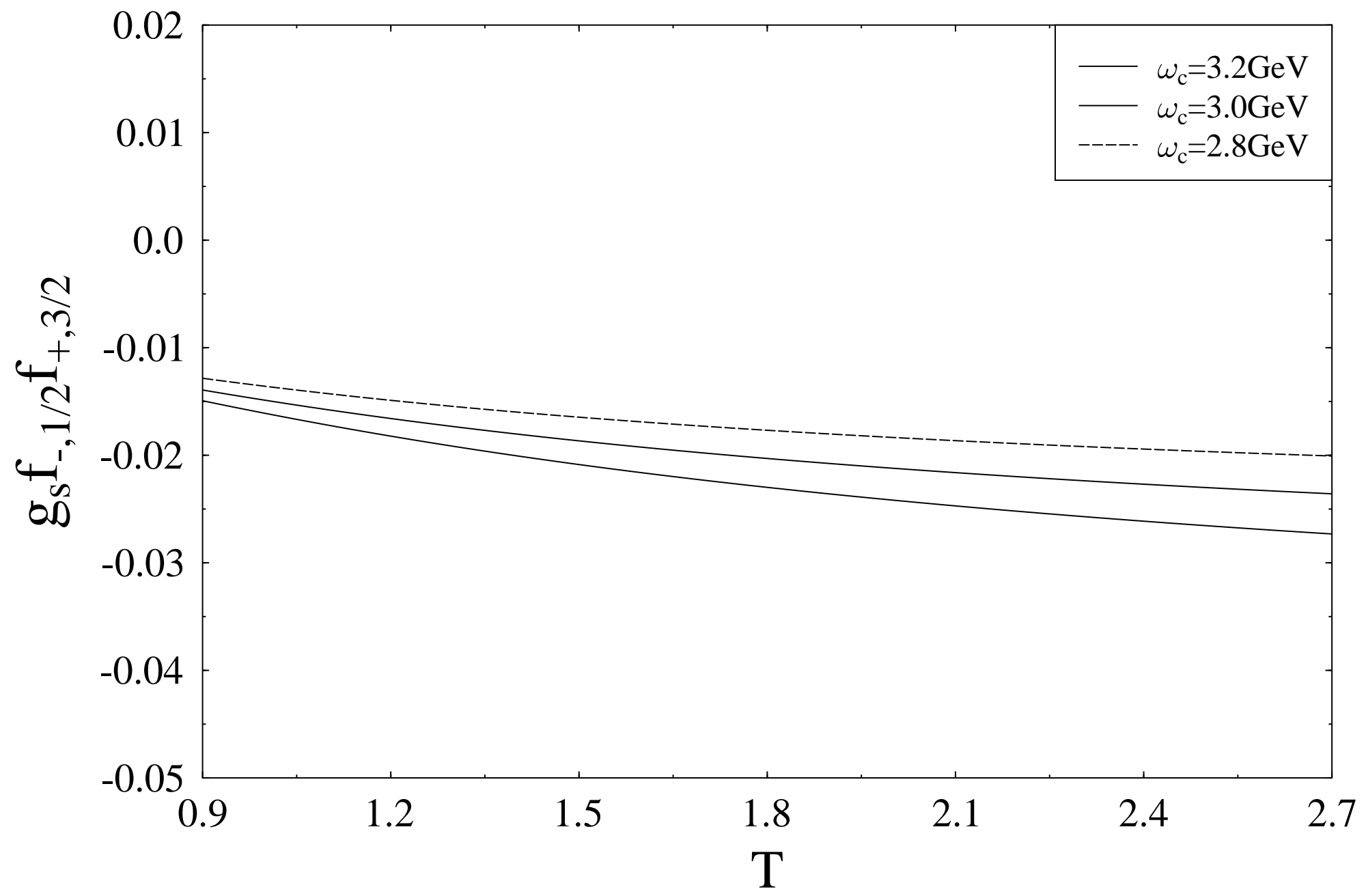




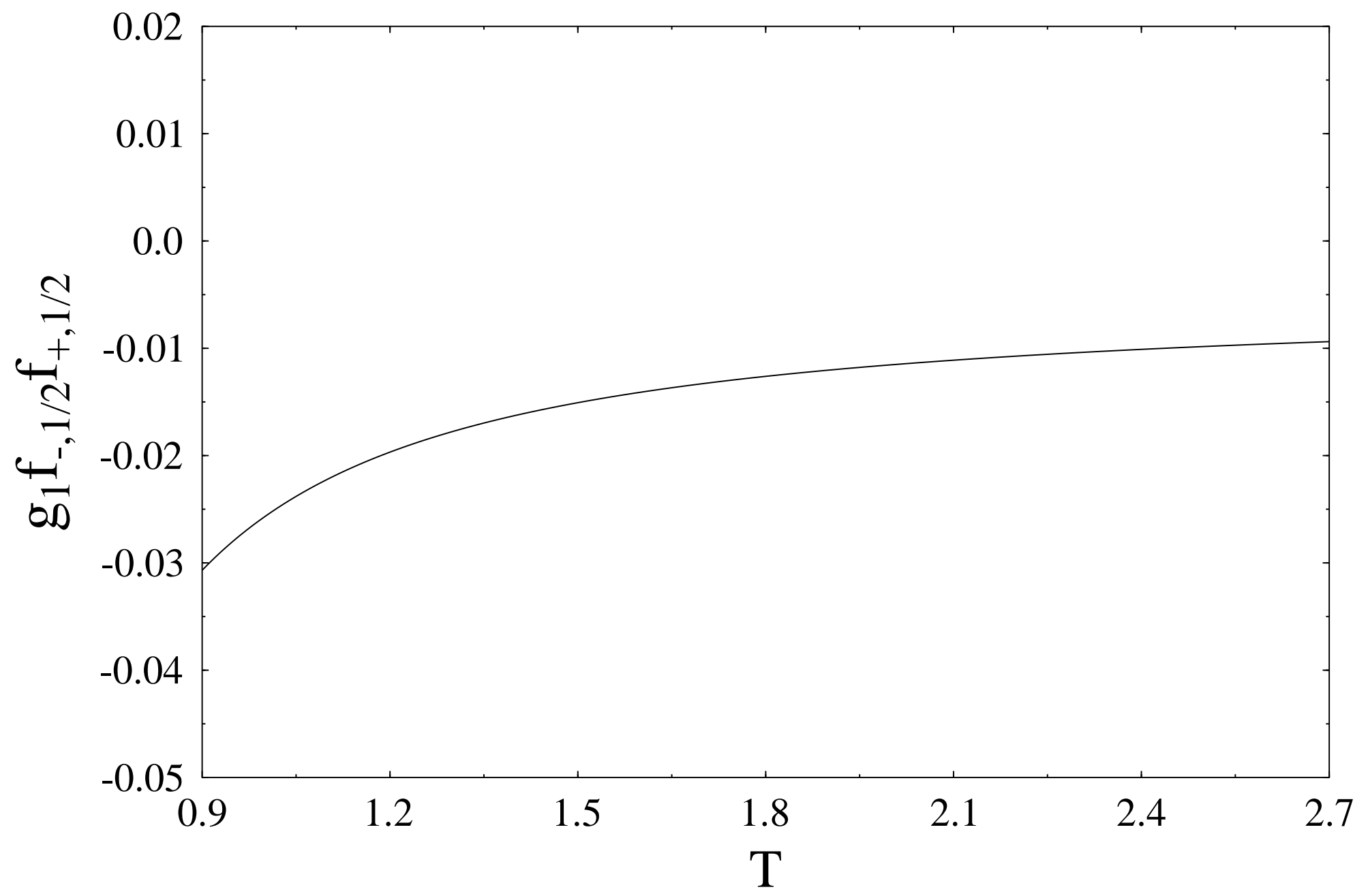




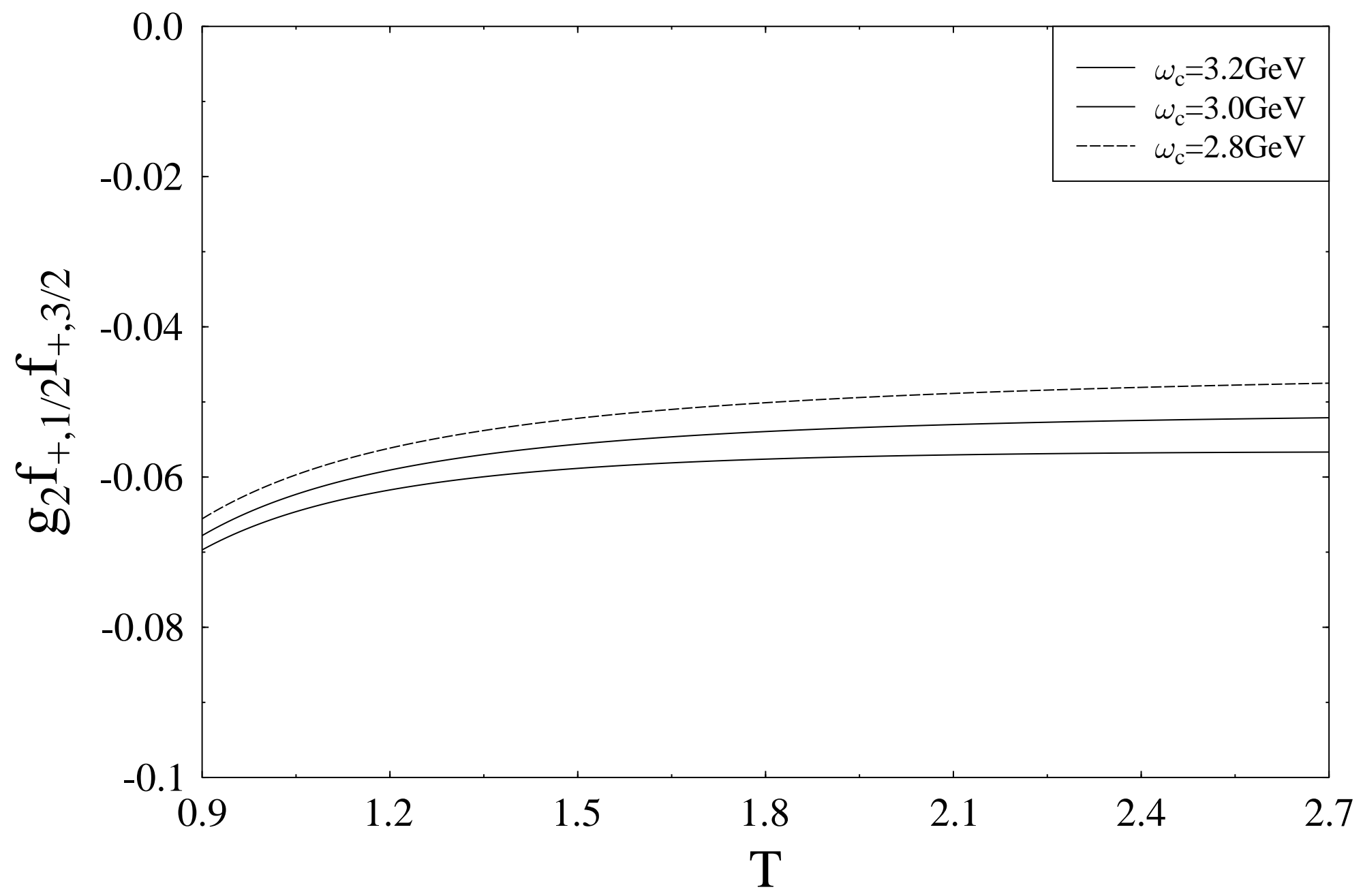




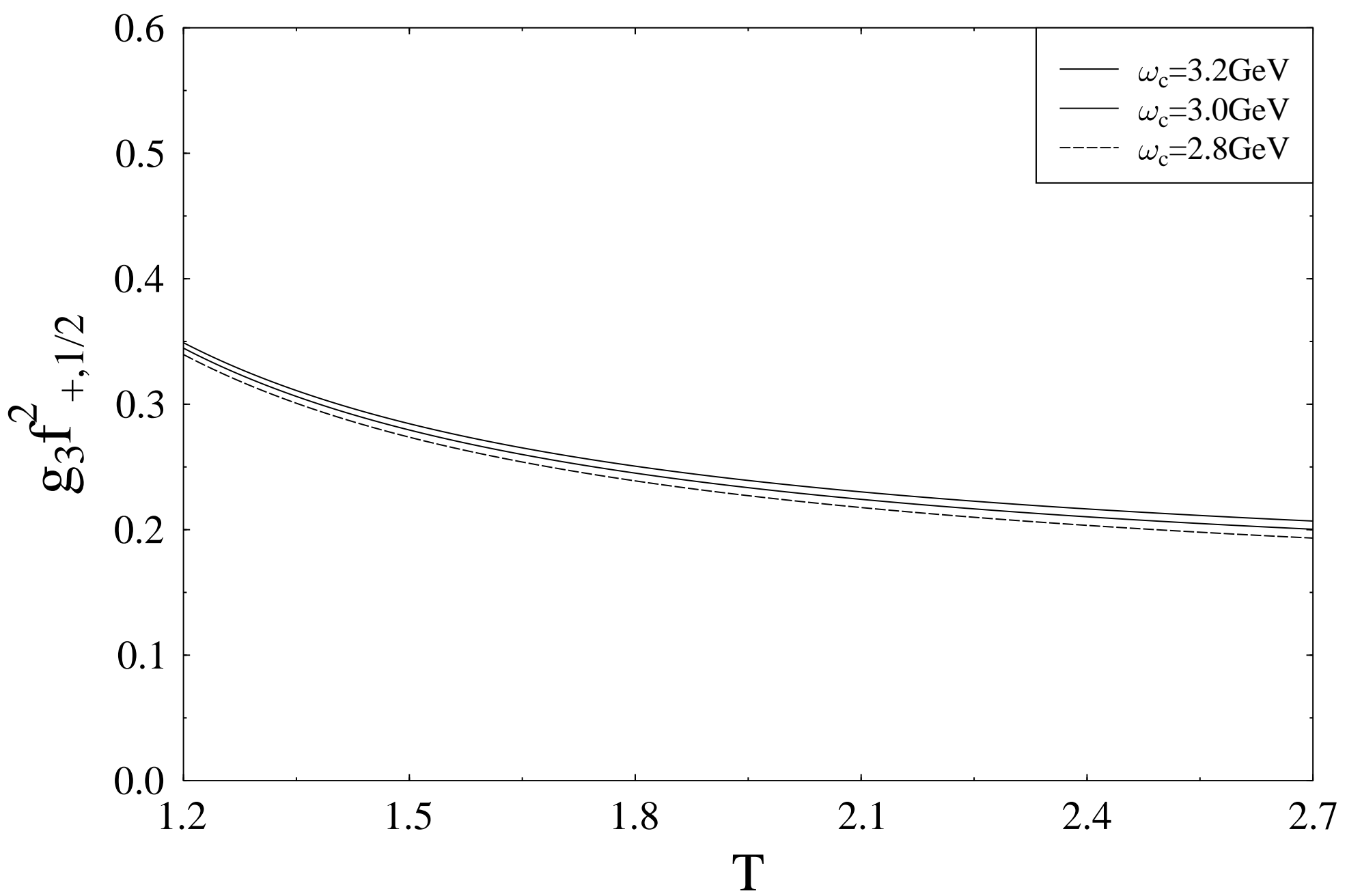




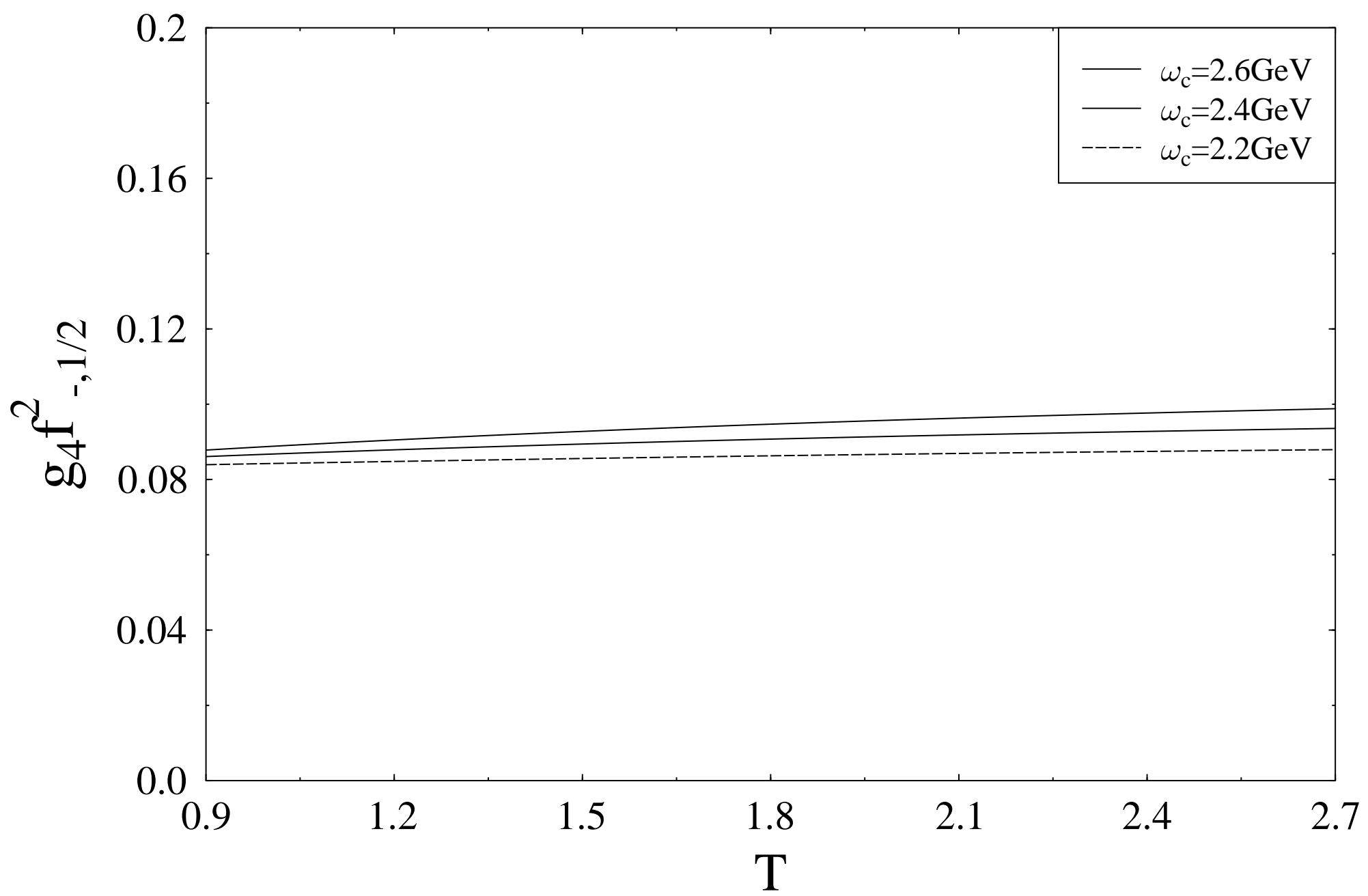




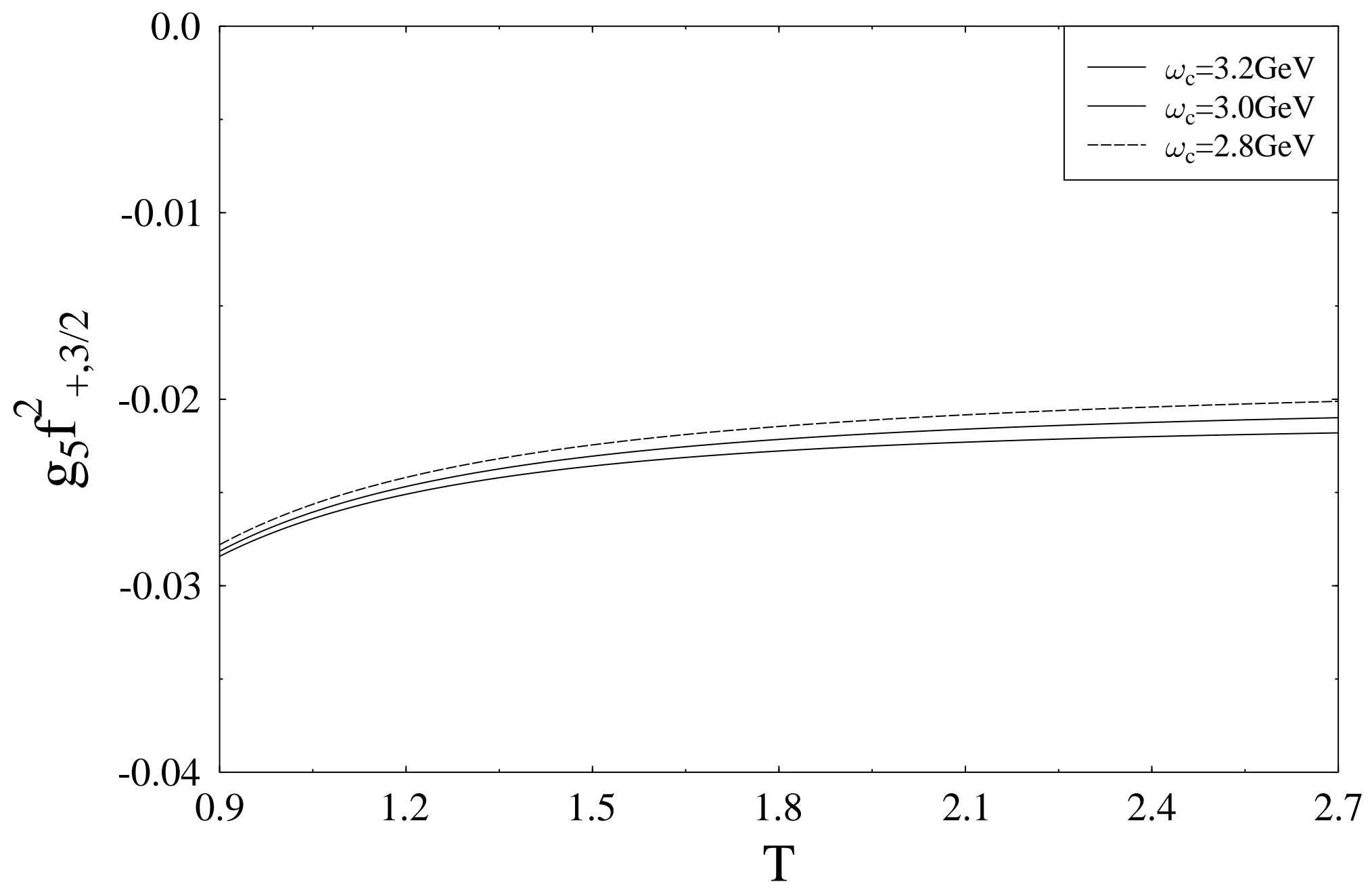

